\title{
不同日照强度下的舰船目标识别
}

\author{
刘坤, 米乐红 ${ }^{*}$ \\ (上海海事大学信息工程学院 上海 201306) \\ (929208009@qq.com)
}

\begin{abstract}
摘 要: 海面目标监测时, 舰船目标的清晰度常随着不同的日照强度下海面光线反射强度而变化, 不同的日照强度 会导致舰船目标识别率不稳定，出现误判虚警率提高等问题. 为此, 提出基于 ResNet-50 的舰船目标识别算法. 首先 使用 ResNet-50 网络提取图像特征信息，并对日照强度变化前后的特征进行日照鲁棒损失约束，减小特征差异; 然后 采用灰度直方图计算特征统计矩的方法得到日照对比度、亮度、平滑度、信息量、三阶矩和熵 6 种特征, 并生成新 的特征向量对日照强度前后的特征再次进行日照鲁棒损失约束，削弱和约束日照变化前后亮度、对比度因素对特征 的影响; 最后将二者约束联合构成损失函数并进行训练, 使用贝叶斯自适应超参数优化训练最佳权重. 实验结果表 明，针对舰船日照变化数据库的平均识别率达到 $90.47 \%$, 比改进前提高 $4.00 \%$ 左右，对日照强度为 25,65 和 80 的舰 船图像识别率分别提高 3.14\%, 6.07\%和 16.41\%, 该算法对日照强度变化有着良好的约束作用, 显著提升了识别率.
\end{abstract}

关键词: 图像识别; 日照变化; 灰度直方图; 贝叶斯超参数优化; 深度学习; 舰船识别; 鲁棒特征损失函数 中图法分类号: TP391.41ＤOI: 10.3724/SP.J.1089.2021.18777

\section{Ship Target Recognition Under Different Sunlight Intensity}

\author{
Liu Kun and Mi Lehong* \\ (College of Information Engineering, Shanghai Maritime University, Shanghai 201306)
}

\begin{abstract}
In the case of surface target monitoring, the clarity of the ship target often varies with the reflection intensity of the sea surface under different sunlight intensity, which will lead to the unstable recognition rate of the ship target and increase the false alarm rate. For this reason, the ship target recognition algorithm based on ResNet-50 is proposed. Firstly, it uses ResNet-50 network to extract image feature information and applies sunlight robust loss constraint to the features before and after sunlight intensity change to reduce the feature difference. Then, it uses gray-scale histogram to calculate the statistical matrices of features to obtain six features: light contrast, brightness, smoothness, information, third-order matrices and entropy, and generates new feature vector to apply sunlight robust loss constraint to the features before and after sunlight intensity change again. Finally, the two constraints are combined to form a loss function and trained to optimize the optimal weights using Bayesian adaptive hyperparameters. The experimental results show that the average recognition rate of the database for ship sunlight variation reaches $90.47 \%$, which is about $4.00 \%$ higher than that before the improvement, and the recognition rate of ship images with sunlight variation of 25, 65 and 80 increases by 3.14\%, 6.07\% and 16.41\%, which shows that the algorithm has a good constraint effect on sunlight variation and the recognition rate is significantly improved.
\end{abstract}

收稿日期: 2020-11-14; 修回日期：2021-06-04. 基金项目：国家自然科学基金(61803257); 航空科学基金(201955015001). 刘坤 (1982一), 女, 博士, 副教授, 硕士生导师, 主要研究方向为模式识别与智能系统、图像处理; 米乐红(1996一), 女, 硕士研究生, 论文 通讯作者, 主要研究方向为遥感图像处理、目标识别. 
Key words: image recognition; illumination variation; gray histogram; Bayesian hyperparameter optimization; deep learning; ship recognition; robust feature loss function

目前, 海上交通防护、海洋环境管理和海域信 息监测等越发重要, 如何有效、准确地识别舰船目 标是目前海洋智能化信息监控的重要研究方向. 传统的船舶识别 ${ }^{[1-4]}$ 主要依据人工设计特征且依赖 手工调试参数, 参数量少, 其中特征提取多以目标 的颜色、纹理、形状、空间、 $\mathrm{Hu}$ 矩特征 ${ }^{[5]}$ 和局部 二值模式(local binary pattern, LBP)特征等为主, 同时, 为了减少误判, 这些设计的特征描述符和分 类器通常非常复杂, 不适用于实时系统.

随着深度学习理论的深人, 许多学者深人开 展了基于深度学习的目标识别、分类以及检测等算 法研究 ${ }^{[6-10]}$. 但受限于数据采集和海面环境复杂等 因素, 基于海面舰船目标识别算法的研究仍相对 有限. 目前国内外基于舰船目标的 HRSC2016, DOTA 等公共数据集被广泛应用于舰船检测，但针 对日照强度不同而设计的数据集相对较少; 同时, 海面气象环境复杂直接干扰图像的采集以及目标 监控，其中自然日照强度是最常见的一种干扰因 素. 因海面光洁平滑易映射太阳，日照强度不同会 使海面上形成不同程度的反光, 阳光的强反光反 射到舰船上形成不同程度的曝光，折射率越大，反 射越剧烈, 反射能流也越大, 这一现象常导致舰船 目标特征信息模糊、缺失或与海面融为一体较难区 分, 进而影响后续的识别和处理.

针对此类日照强度不同影响舰船识别率的问 题, 刘欢等 ${ }^{[11]}$ 在 $\mathrm{Hu}$ 不变矩的基础上, 重新组合构 造出新组合不变矩并将其应用到图像识别中，其 具有良好的性能, 包括光照鲁棒性和模糊稳定性, 针对受光照变化和模糊噪声一致作用造成的识别 率下降问题, 提出了组合不变矩图像匹配方法. Zhuang 等 ${ }^{[12]}$ 提出了基于深度学习的方法, 以解决 光照因素对舰船识别 ${ }^{[13]}$ 效果的不利影响, 先进行 光照预处理, 再利用 Log-Gabor 滤波器获取特征图 像, 提取图像子块的 LBP 特征形成纹理特征直方 图, 并将其输人深度信念网络(deep belief networks, $\mathrm{DBN})^{[14]}{ }^{[1}$ 视觉层中. 但目前常用的方法仍倾向于用 不变矩以及图像预处理，易忽略目标局部特征.

如何有效地抑制因日照强度不同而导致的舰 船目标识别率不稳定以及误判, 是目前亟待解决 的问题. 针对这一问题, 本文开展了基于卷积神经 网络的舰船目标鲁棒特征的识别算法研究. 首先, 使用 ResNet-50 网络提取图像特征信息; 然后, 使
用日照鲁棒损失函数使不同日照强度与清晰样本 图像特征差异最小化; 同时, 使用灰度直方图计算 特征统计矩的方法生成 6 维特征向量, 对不同日照 强度与清晰样本特征差再次进行日照鲁棒损失函 数约束, 减小光照的影响; 最后, 使用贝叶斯自适 应超参数优化训练得到识别率最高的权重分配. 实验结果表明，本文算法抑制了不同光照对舰船 目标识别准确性的干扰, 平均识别率可达 $90.47 \%$. 该算法不仅可应用于海面监控监测，也可应用于 海防预警、侦察识别等领域.

\section{1 本文算法}

\section{1 建立舰船图像数据库}

目前针对日照强度不同所构成的公共舰船数 据集相对较少, 本文的数据库首先在谷歌地图上 收集了不同时间、不同日照强度的真实舰船图像 12000 幅. 真实数据集中有清晰图像和因日照反 光、鱼鳞光和海面杂波影响图像特征的舰船图像, 是同一场景不同日照强度下获取的不同图像. 真 实图像因日照强度不同会导致舰船信息不全甚至 缺失, 如图 1a 中舰船目标在强阳光照射下航行时, 大面积的强光反射干扰致船身无法清晰可见; 图 1b 中常见的海面鱼鳞光使舰船目标受到大面积的 海面杂波干扰; 图 1c 为舰船航迹引起的海浪和反 射光照射到船身使海浪与船身不易分离.

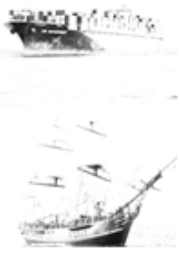

a. 强光反射

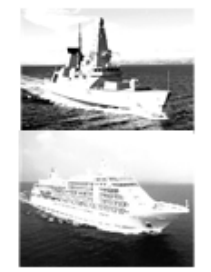

b. 鱼鳞光杂波干扰

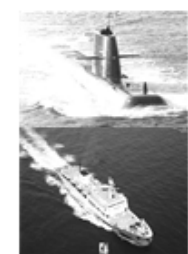

c. 航迹
图 1 真实曝光的部分舰船目标

由于与日照强度相关的数据集不足, 因此对 部分舰船图像做人工日照处理, 通过伽马校正 ${ }^{[15]}$ 图像亮度, 在输人值和映射的输出值之间使用非 线性变换, 并通过数据增强得到模拟图像, 且让图 像本身的曝光位置随机分布. 本文将模拟和真实 图像一起作为训练数据来进行实验. 其中, 从真实 图像中取 6000 幅 7 类舰船样本用来人工模拟日照, 
使用伽马校正方法构建模拟不同日照强度数据集, 包括航空母舰 900 幅、游艇 1000 幅、驱逐舰 900 幅、渔船 800 幅、帆船 800 幅、潜水艇 800 幅、运 输船 800 幅. 经处理后的图像 30000 幅, 加上真实 数据集共 42000 幅, 按照日照强度顺序增强排列, 选择真实数据集 4200 幅和人工模拟图像 25800 幅 用于训练，其余部分 $1: 1$ 用于验证和测试，训练、 验证和测试图像数量比例为 $5: 1: 1$. 将所有图像 经过预处理调整为尺寸为 $224 \times 224 \times 3$ 的样本, 图 2 所示为不同日照强度的舰船识别部分样本.

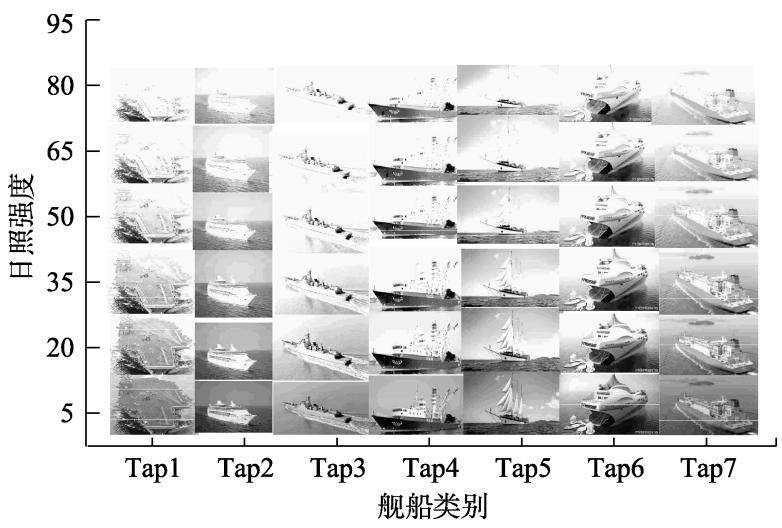

图 2 模拟不同日照强度的部分舰船图像

伽马校正方法可以改变图像亮度，构造出同一 图像在不同日照强度下的效果. 设置 $K$ 个日照强度 变化系数 $\psi=\left\{\psi_{1}, \psi_{2}, \psi_{3}, \cdots, \psi_{k}\right\}$ 以及对应的变换 $T_{\psi /}=\left\{T_{\psi_{1}}, T_{\psi_{2}}, T_{\psi_{3}}, \cdots, T_{\psi_{k}}\right\}$, 对模拟训练样本 $X=\left\{X_{1}\right.$, $\left.x_{2}, x_{3}, \cdots, x_{N}\right\}$ 进行变化变换 $T_{\psi}$, 可以得到 $K$ 倍的样 本数据, 且变换过后的训练样本为 $T_{\psi} X=\left\{T_{\psi} X_{1}\right.$, $\left.T_{\psi} x_{2}, T_{\psi} x_{3}, \cdots, T_{\psi} x_{N}\right\}$. 其中, 单个样本经过变化变换 后的新样本为 $T_{\psi} x_{i}=\left\{T_{\psi_{k}} x_{i} \mid k=1,2,3, \cdots, k\right\}$, 原始样 本和变化样本共同构成模拟训练样本。在本文实 验中, 设 $K=5$, 日照强度 $\psi=\{10,25,50,65,80\}$, 通
过设置不同的伽马值构造与日照强度对应的图像, 同一幅图像可生成 5 幅不同日照程度的舰船图像. 可对某部分像素进行设置, 使用均匀分布选择像 素是为了保证日照方向以及部位的随机性. 让曝 光模块以一定概率随机均匀地分布整幅图像，以 便生成不同日照强度的图像, 避免同一位置的日 照强度使实验结果缺少泛化能力.

\section{2 基于 ResNet 的舰船识别网络框架}

2016 年, He 等 ${ }^{[16]}$ 发现的深度残差网络 ResNet 在 ImageNet 上图像分类识别 Top-5 达到 3.57\%. ResNet 参数量低, 它直接将输人特征通过残差模 块传到输出信息, 保证特征信息的完备性, 解决了 信息遗失、耗损等问题，避免梯度消失或梯度爆炸. 整个 ResNet 只需学习输人信息和输出的残差, 可 以很快地加速神经网络的运行, 简化了学习目标 和难度. 本文针对不同日照强度环境下舰船识别 率不稳定的问题, 提出基于 ResNet 的舰船目标特 征鲁棒的识别算法, 其网络框图流程如图 3 所示, 算法网络结构为 51 层.

首先，将尺寸大小为 $224 \times 224 \times 3$ 的舰船图像 输人, 经过 64 个卷积核为 $7 \times 7$ 且步长为 2 的卷积 层, 得到 $112 \times 112 \times 64$ 的特征图; 然后, 再通过步长为 2 的最大池化, 得到尺寸为 $56 \times 56 \times 64$ 的池化特征, 并 将该特征依次经过 16 个残差模块得到 $7 \times 7 \times 2048$ 的 特征图, 再通过平均池化得到 $1 \times 1 \times 2048$ 的特征向 量; 然后将该特征向量经过日照鲁棒损失函数进 行约束, 使不同日照强度与原始日照强度的差异 最小化，这是抑制日照下舰船目标特征缺失及模 糊的关键函数; 最后, 将约束后的特征向量送人尺 寸为 $(7,2048)$, 全连接层后用 Softmax 函数进行分 类识别舰船图像，其中有航空母舰、游艇、驱逐舰、 渔船、帆船、潜水艇和运输船等 7 类目标, 分别用 Tap1 Tap7 表示.

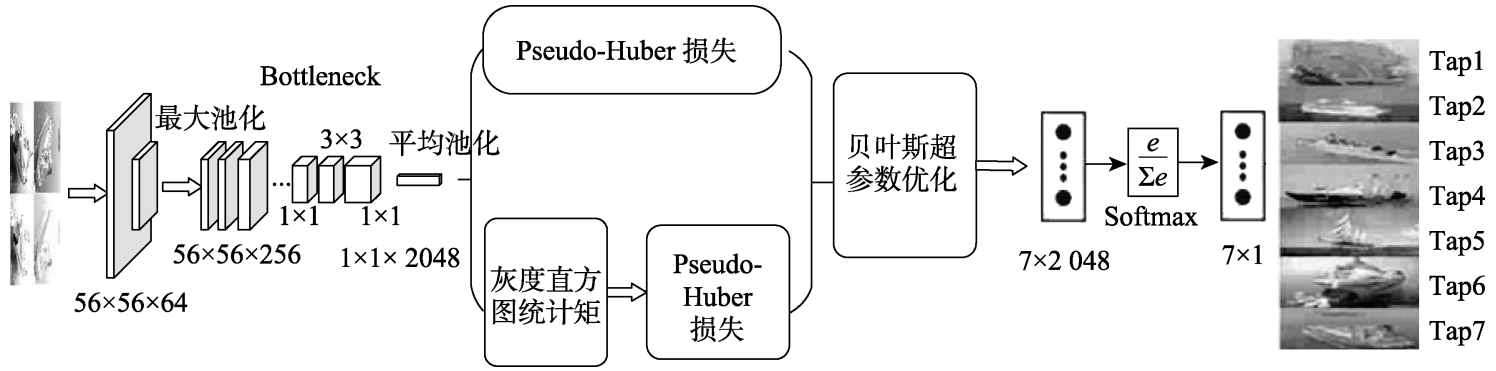

图 3 舰船目标特征不变的识别方法框架图

对目标函数使用 Adam 算法 ${ }^{[17]}$ 进行训练, 训 练过程包括前向传播和反向传播. 计算目标值与
实际输出偏差，再利用偏差范围反向传播更新权 值和参数; 优化时采用贝叶斯超参数优化方法对 
权重进行自适应优化，建立代理函数代替目标函 数, 通过评估代理函数上超参数的性能, 为目标函 数提供超参数的最优组合, 寻找最佳权重衡量鲁 棒特征损失函数项之间的关联; 对比人工调参, 自 适应优化后的权重更利于提高识别率. 选取不同类 别、不同日照强度舰船图像各 500 幅进行测试, 比 较预测值与真实值, 计算网络模型的平均识别率.

\section{2 日照强度不同的特征鲁棒性分析}

\section{1 设计鲁棒性特征损失函数}

日照强度不同图像使卷积神经网络特征提取 过程中缺失信息, 学习特征不充分会导致识别过 程中错误率上升. 为改善此种现象, 本文提出的不 同日照强度的鲁棒性特征损失函数包含 2 部分, 一 是利用 Pseudo-Huber 损失函数 ${ }^{[18]}$ 减小不同日照强 度图像与清晰图像的差异, 使缺失的特征不会影 响分类器的识别功能, 提高识别的精确度; 二是将 不同日照强度图像经过网络最后一层平均池化输 出的特征向量提取灰度级特征, 计算统计矩(均 值、标准方差、平滑度、三阶矩、一致性和熵), 合 成新特征向量. 再次利用 Pseudo-Huber 损失函数 针对亮度对比度因素影响进行约束, 以此抑制日 照对识别率的影响. Pseudo-Huber 损失是 Huber 损 失的连续光滑逼近, 确保了所有角度上是连续可 导的. Pseudo-Huber 函数定义为

$$
L_{\delta}(a)=\delta^{2}\left(\sqrt{1+\left(\frac{a}{\delta}\right)^{2}}-1\right)
$$

其中, $a$ 为日照强度 $10,25,50,65,80$ 舰船的特征向 量平均值与未经过日照处理清唽样本的特征差; $\delta$ 为超参数. 首先, 度量不同日照强度舰船图像与 清晰图像的特征差. 在不同日照强度舰船识别算 法中, $\boldsymbol{O}\left(x_{i}\right)$ 和 $\boldsymbol{O}\left(T x_{i}\right)$ 分别为清晰图像与日照处理 后图像经过最后一层平均池化后的特征向量, $\overline{\boldsymbol{O}\left(T x_{i}\right)}$ 代表日照强度分别为 $10,25,50,65,80$ 经过 ResNet-50 最后一层平均池化的特征向量的平均 值. 将 $\boldsymbol{O}\left(x_{i}\right)$ 和 $\boldsymbol{O}\left(T x_{i}\right)$ 分别代人式(1)建立特征约束 函数

$$
d\left(\boldsymbol{O}\left(x_{i}\right)-\overline{\boldsymbol{O}\left(T x_{i}\right)}\right)=\delta^{2}\left(\sqrt{1+\left(\frac{\boldsymbol{O}\left(x_{i}\right)-\overline{\boldsymbol{O}\left(T x_{i}\right)}}{\delta}\right)^{2}}-1\right)
$$

通过减小日照强度变化前后的特征差，提高特征 提取鲁棒性.

同时, 考虑光照因素影响，提取舰船图像通过
最后一层池化层后的特征向量计算灰度直方图, 和 统计矩合成新特征向量. 再次利用 Pseudo-Huber 损 失函数削弱日照对识别率的影响, 分别计算全连 接层前尺寸大小为 $1 \times 1 \times 2048$ 的特征向量灰度级特 征和统计矩, 包括均值 $m$ 、标准方差 $\sigma$ 、平滑度 $R$ 、 三阶矩 $u_{3}$ 、一致性 $U$ 以及熵 $e$, 组成新的特征向量 $\boldsymbol{O}_{\mathrm{FC}}(x)=\left(m, \sigma, R, u_{3}, U, e\right)$, 如

$$
\left\{\begin{array}{l}
m=\sum_{i=0}^{l-1} z_{i} p\left(z_{i}\right) \\
\sigma=\sqrt{\sum_{i=1}^{l-1}\left(z_{i}-m\right)^{2} p\left(z_{i}\right)} \\
R=\frac{1}{1+\sigma^{2}} \\
u_{3}=\sum_{i=1}^{l-1}\left(z_{i}-m\right)^{3} p\left(z_{i}\right) \\
U=\sum_{i=0}^{l-1} p^{2}\left(z_{i}\right) \\
\left.e=-\sum_{i=0}^{l-1} p\left(z_{i}\right) \operatorname{lbp} p\left(z_{i}\right)\right)
\end{array}\right.
$$

再代人 Pseudo-Huber 损失函数进行特征约束, 以 减小光照对识别率的影响. 其中, $L$ 为灰度级总 数; $z_{i}$ 表示第 $i$ 个灰度级; $p\left(z_{i}\right)$ 为归一化直方图灰 度级分布中灰度为 $z_{i}$ 的概率.

$\boldsymbol{O}_{\mathrm{FC}}\left(x_{i}\right), \boldsymbol{O}_{\mathrm{FC}}\left(T x_{i}\right)$ 是清晰图像与日照处理后的 图像在平均池化后提取灰度级特征计算统计矩后 生成的新特征向量, 将它们的平均值分别代人式 (1), 建立特征约束函数

$$
\begin{aligned}
& d\left(\boldsymbol{O}_{\mathrm{FC}}\left(x_{i}\right)-\overline{\boldsymbol{O}_{\mathrm{FC}}\left(T x_{i}\right)}\right)= \\
& \delta^{2}\left(\sqrt{1+\left(\frac{\boldsymbol{O}_{\mathrm{FC}}\left(x_{i}\right)-\overline{\boldsymbol{O}_{\mathrm{FC}}\left(T x_{i}\right)}}{\delta}\right)^{2}}-1\right)
\end{aligned}
$$

通过减小光照、亮度、信息量相关的向量特征与原 图像的差距, 减弱不同日照强度对识别的影响, 提 高对舰船的识别率.

本文算法总损失函数包括分类交叉熵损失和 鲁棒特征约束函数, 如

$$
\begin{gathered}
L=\frac{-1}{N K+M} \sum_{k=1}^{C} y_{\left(x_{i}\right)}[k] \log \frac{\mathrm{e}^{\left(\boldsymbol{W}_{y_{i}}^{\mathrm{T}} x_{i}+b_{y i}\right)}}{\sum_{j=1}^{n} \mathrm{e}^{\left(\boldsymbol{W}_{y_{j}}^{\mathrm{T}} x_{j}+b_{y j}\right)}}[k]+ \\
\lambda_{1} d\left(\boldsymbol{O}\left(x_{i}\right)-\overline{\boldsymbol{O}\left(T x_{i}\right)}\right)+\lambda_{2} d\left(\boldsymbol{O}_{\mathrm{FC}}\left(x_{i}\right)-\overline{\boldsymbol{O}_{\mathrm{FC}}\left(T x_{i}\right)}\right)
\end{gathered}
$$

其中, $C$ 为舰船类别数; $y_{\left(x_{i}\right)}$ 为样本的真值标签向 量; $N$ 为采用模拟舰船数; $K$ 为日照强度变化系 数; $M$ 为真实舰船数. 第 1 项为交叉熵损失, 用来 分类识别; 第 2 项为 Pseudo-Huber 损失函数约束; 第 3 项为灰度直方图统计矩约束损失项; 后 2 项约 
束了特征间的差异， $\lambda_{1}$ 和 $\lambda_{2}$ 为权重系数, $\boldsymbol{W}$ 为权 值矩阵, $b$ 为偏置量. $\lambda_{1}$ 和 $\lambda_{2}$ 使用贝叶斯自适应 超参数算法调参 ${ }^{[19]}$, 具体详见实验结果与分析. 鲁棒损失函数加人深度学习的训练过程本质是使 梯度下降, 减小损失. 在训练过程中减小上述特征 差,使日照前后特征差减小，削弱光照因素影响， 从而提高识别率.

\section{2 日照强度不同的特征分析}

对比不同日照强度经损失函数约束后的变化, 对比本文的鲁棒特征损失函数与 4 种常用约束函 数. 网络模型依旧以本文基于 ResNet 的识别算法,
考虑损失函数和超参数的影响, 分别与 $L_{1}, L_{2}$ 损失 进行对比, 并且考虑无约束、单约束和双约束的情 况. 4 种约束函数分别为 $L_{1}$ 损失函数 (ResNet- $\left.L_{1}\right)$, $L_{2}$ 损失函数 (ResNet- $L_{2}$ ), Pseudo-Huber 损失函数 (ResNet-hb) 和提取特征向量灰度直方图, 并计算 统计矩生成新特征向量后, 再进行 Pseudo-Huber 损失函数约束方法 (ResNet-sm). 分别选取日照强 度为 $0,10,25,50,65,80$ 的不同类舰船图像各 20 幅, 分别比较各日照强度下的样本目标与日照强 度为 0 的标准样本经过全连接层输出的特征向量 差的平均方差, 如表 1 所示.

表 1 不同日照强度下与清晰样本舰船特征差的平均方差对比

\begin{tabular}{cccccc}
\hline 日照强度 & ResNet- $L_{2}{ }^{[16]}$ & ${\text { ResNet- }{ }_{1}{ }^{[14]}}$ & ResNet-hb $^{[18]}$ & ResNet-sm $^{[20]}$ & 本文 \\
\hline 10 & 0.63 & 0.66 & 0.24 & 0.11 & 0.03 \\
25 & 0.85 & 0.97 & 0.43 & 0.28 & 0.09 \\
50 & 1.06 & 1.13 & 0.60 & 0.42 & 0.18 \\
65 & 1.24 & 1.22 & 0.80 & 0.56 & 0.24 \\
80 & 1.53 & 1.51 & 1.00 & 0.70 & 0.30 \\
\hline
\end{tabular}

在日照强度较强时, ResNet- $L_{1}$ 比 ResNet- $L_{2}$ 的 平均方差小, 效果更优, 这是由于 ResNet- $L_{1}$ 处理 异常点时更稳定, 性能也较为鲁棒. 而在日照强度 较弱时, ResNet- $L_{2}$ 比 ResNet- $L_{1}$ 鲁棒性更高, ResNet- $L_{1}$ 损失更新的梯度始终相同，即使对于很小的 损失值梯度相对也很大，但 ResNet- $L_{2}$ 损失使用固定 的学习率也可使损失函数有效地收玫. Pseudo-Huber 损失函数综合 $L_{1}$ 损失函数和 $L_{2}$ 损失函数的优点, 既稳定处理异常点, 又确保导数可导. 从表 1 可以 看出, ResNet-hb 和 ResNet-sm 的平均方差较小, 在 不同日照强度下都能保证舰船特征的相似性; 将 2 种约束结合的本文算法平均方差最小, 不同日照强 度的舰船图像与清晰图像的差异较小, 稳定性强.

为进一步验证鲁棒特征损失函数对不同日照 强度下舰船的特征约束性能, 选取游艇舰船图像 以及经过日照强度 $10,25,50,65,80$ 处理后的图像 经过 ResNet 平均池化, 得到 2048 维特征向量, 并 进行主成分分析(principal component analysis, PCA) 降维得到 64 维主特征; 分别计算日照处理图像与 清晰样本的特征差, 并与鲁棒特征损失函数约束 后的特征差进行对比, 以直观呈现本文中约束的 效果.

表 2 体现出经过不同日照强度的舰船图像在 经过鲁棒特征损失函数约束之后的特征差的区别. 表 2 中每列分别为不同算法在不同日照强度下的
特征差. 其中, 第 2 列为原始数据的特征差, 即 $\lambda_{1}=\lambda_{2}=0$; 第 3 列为 ResNet-hb 的舰船图像特征 差, $\lambda_{1}>0, \lambda_{2}=0$; 第 4 列为 ResNet-sm损失约束后 的舰船特征差, $\lambda_{1}=0, \lambda_{2}>0$; 第 5 列为本文算法 舰船特征差, $\lambda_{1}>0, \lambda_{2}>0$. 可以看出, 日照强度 的不同会对图像特征产生影响, 且变化程度越大, 特征差越大, 特征的差异性会使舰船识别更加困 难. 经过 ResNet-hb 使变化前后图像特征差有所收 玫, 日照强度 50,65, 80 的图像特征差的极差分别 从为 $3.60,4.80,6.00$ 下降到了 $1.20,1.60,2.00$, 减 小了约 67\%; 而 ResNet-sm 在日照亮度、对比度和 平滑度等对特征的约束下，使特征差的极差下降 为 $0.84,1.12,1.40$, 比约束前平均减小了 $77 \%$. 从 日照的亮度对比度分析, ResNet-sm 对特征的约束 力度大, 其与 ResNet-hb 相比提升了识别率; 对日 照强度为 $50,65,80$ 舰船特征, 本文算法约束后的 特征差的极差分别下降到 $0.36,0.48,0.60$. 可明显 看出, 采用 2 个共同约束减小了变化前后的图像特 征差异, 提高了识别率, 增加鲁棒性. 实验验证了 ResNet-hb 和 ResNet-sm 对变化特征的约束作用, 而且两者的共同约束会使图像特征差更小.

为进一步验证联合约束的高鲁棒性, 选取日 照强度分别为 50,80 的不同类舰船图像各 50 幅经 过 ResNet 最后一层平均池化, 得到 2048 维特征向 量, 并进行 PCA 降维得到 64 维主特征, 作出特征 
表 2 不同日照强度下鲁棒特征损失函数下的特征差折线图对比

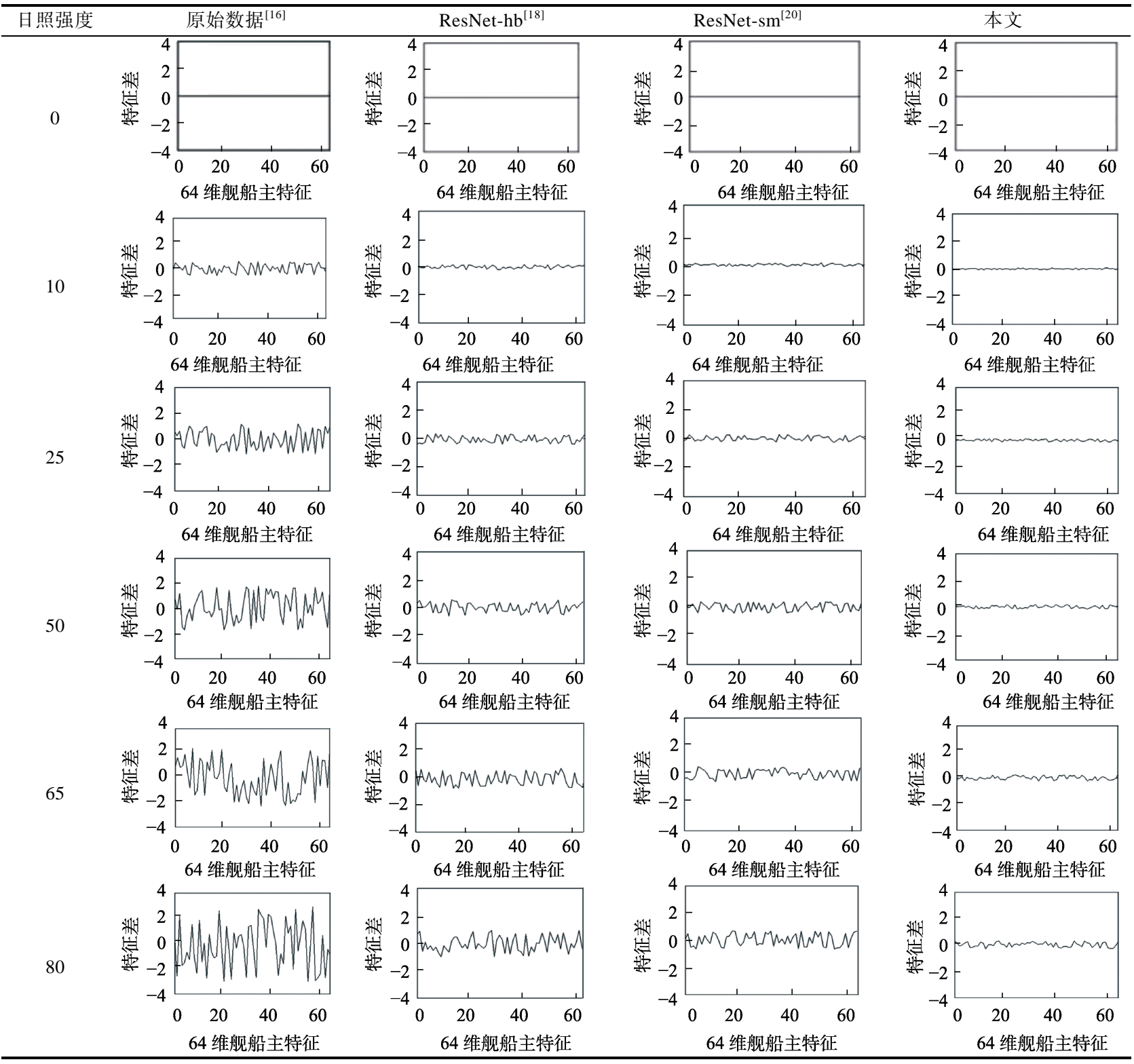

直方图. 计算相同日照条件下不同类舰船与清晰 图像直方图的平均巴氏系数 ${ }^{[21]}$, 对相同的数据点 乘积开方并累加得到的结果为相似度值，即巴氏 系数, 如表 3 所示. 表 4 所示为部分游艇图像的直 方图. 其中, ResNet-hb 的直方图变化较明显, 且日 照强度 50 的不同类舰船与清晰图像的平均巴氏系 数为 0.87 , ResNet-hb 减小了日照强度不同时舰船 图像的形状和纹理差异; ResNet-sm 的直方图有轻 微变化, 从图像灰度特征的亮度和对比度来约束 不同日照强度舰船图像，且日照强度为 80 的不同 类图像与清晰图像的平均相似度值达到 0.89 ; 本 文算法的舰船图像在不同日照强度下的直方图几 乎没有变化, 日照强度为 80 的不同类图像与清晰 图像的巴氏系数因子值高达 0.96 , 说明联合约束
下的鲁棒特征损失函数从形状、纹理、亮度和对比 度等各方面进行差异度量, 减少了不同日照强度 下的舰船特征差异，有效地提高了识别率.

表 3 不同日照强度图像约束后相似性度量(巴氏系数)

\begin{tabular}{cccc}
\hline 日照强度 & ResNet-hb $^{[18]}$ & ResNet-sm $^{[20]}$ & 本文 \\
\hline 50 & 0.87 & 0.91 & 0.98 \\
80 & 0.84 & 0.89 & 0.96 \\
\hline
\end{tabular}

由于纹理是由灰度分布在空间位置上反复出 现而形成的，在图像空间中相隔某距离的 2 个像 素之间会存在灰度的空间相关特性. 灰度共生矩 阵 $^{[20]}$ (gray-level co-occurrence matrix, GLCM) 就是 一种通过灰度的空间相关特性描述纹理的常用方 法. 表 5 所示为取日照强度为 80 的不同类舰船图 
表 4 日照强度分别为 50,80 的游艇图像在特征约束下的特征直方图
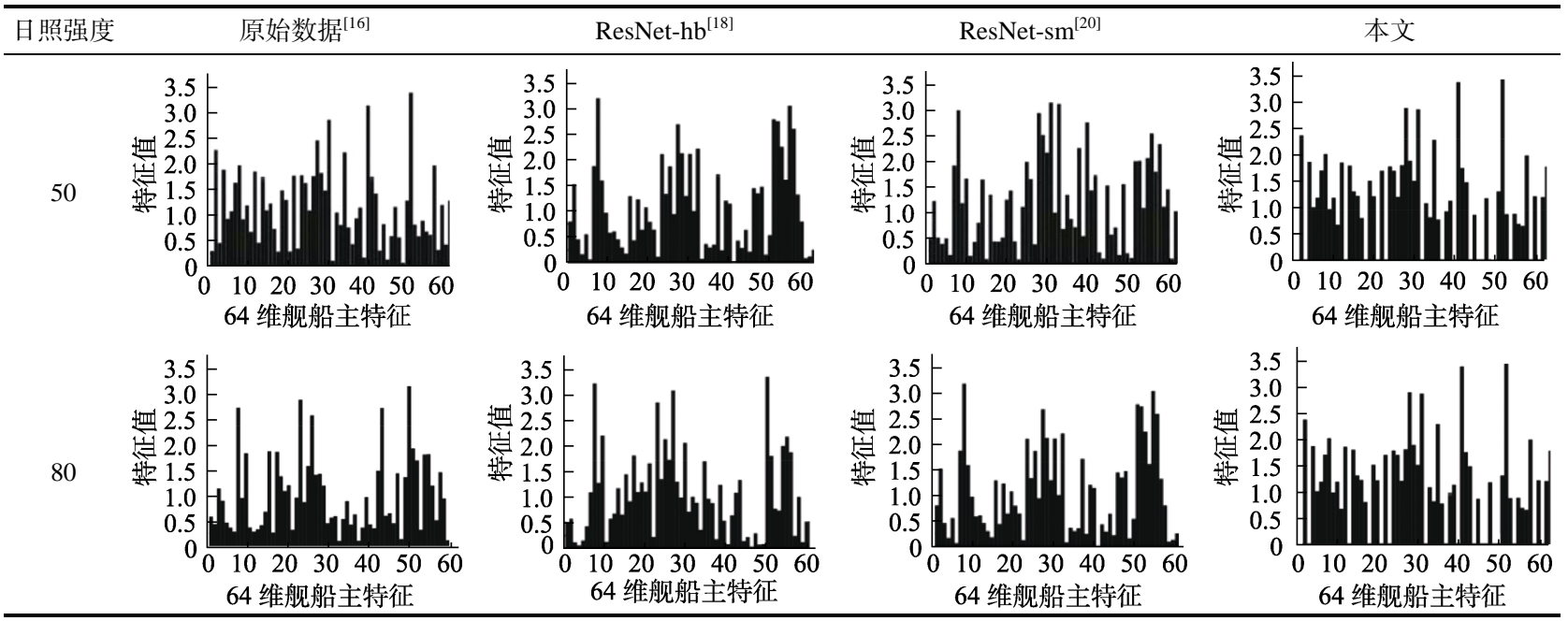

像各 30 幅, 将未经过鲁棒特征损失函数以及经过 损失函数后的全连接层特征向量计算 GLCM, 然 后分别计算 GLCM 的熵、平均对比度、能量、熵 值、相关性和逆方差, 用于度量日照强度不同舰船 特征差异性. 日照强度越高, 与清晰样本的差异越 大. 可以看出, 经过约束之后的对比度提高, 说明 特征纹路沟纹深, 效果清晰; 能量降低说明纹理细 致; 相关性高说明特征越相似; 逆方差大说明纹 理不同区域间较均匀, 变化缓慢. 以上均说明本文 鲁棒特征损失函数对不同日照强度的舰船图像特 征进行了有效约束, 对日照强度有抑制作用.

表 5 日照强度为 80 的不同类舰船图像前后特征差异

\begin{tabular}{lccccc}
\hline \multicolumn{1}{c}{ 算法 } & 对比度 & 能量 & 熵 & 相关性 & 逆方差 \\
\hline ResNet $^{[16]}$ & 0.596 & 0.548 & 0.561 & 0.621 & 2.969 \\
ResNet-hb $^{[18]}$ & 0.693 & 0.369 & 0.492 & 0.795 & 3.216 \\
ResNet-sm & & & \\
吕] & 0.769 & 0.352 & 0.426 & 0.841 & 3.312 \\
& 0.986 & 0.136 & 0.263 & 0.989 & 4.562 \\
\hline
\end{tabular}

\section{3 实验结果与分析}

\section{1 网络训练}

本文的实验环境为 Intel i5-9 4006 核 6 线程，显 卡 GPU 为 GTX1650, 开发平台框架使用 Tensorflow. 学习率设置为 0.010 , 将真实与模拟图像整体作为 训练集, Batch_Size 为 6, 图像按日照强度顺序输 人网络, 每 6 幅图像进行一次提取训练, 再进行迭 代. 设置 $\delta=0.01$, 使用 Adam 算法进行优化. 将损 失函数加人深度学习的训练过程的本质是梯度下 降, 减小损失, 在训练过程中减小特征差, 使日照 前后特征差减小, 削弱光照因素影响, 从而提高识
别率. 计算估计值与实际值之间的误差, 网络训练 反向传播过程是将该误差从输出层向隐藏层反向 传播, 直至传播到输人层, 在反向传播的过程中, 根据误差调整各种参数的值. 不断迭代上述过程, 直至收玫. 本文算法的模型包含 2 个超参数 $\lambda_{1}, \lambda_{2}$, 即神经网络损失函数中权重的分配. 使用贝叶斯 自适应超参数算法调参. 首先, 根据历史验证集合 找出一个可能使损失函数小的超参数集合 $\lambda_{1}, \lambda_{2}$; 其次, 计算损失函数的值, 更新从超参数到损失函 数的映射概率模型——树形估计模型(tree-structured parzen estimator, TPE). 优化准则为预期改进法 (expected improvement, EI), 根据代理函数选择新 的超参数集合 $\lambda_{1}, \lambda_{2}$ 进行实验, 达到迭代次数停止 训练.

本文使用贝叶斯超参数优化对 2 个权重选取 最适宜、使识别率最高的值, 图 4 所示为贝叶斯自 适应超参数训练图. 在迭代的过程中, 不断减小约 束函数值, 使曝光前后的特征最小化, 从而提高识 别率. 在损失函数不断减小的情况下, 超参数在最

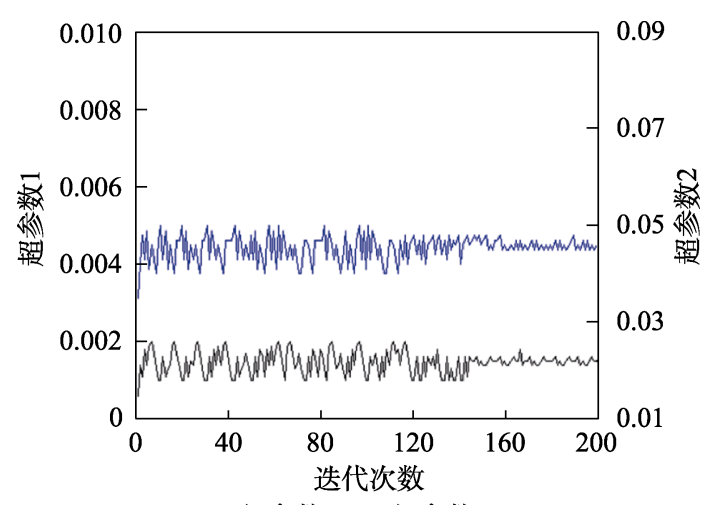

一超参数 1 ; 一 超参数 2

图 4 贝叶斯自适应超参数训练图 
后趋近于 $\lambda_{1}=0.0015, \lambda_{2}=0.0460$ 不变, 是最适宜 权重.

贝叶斯超参数优化有几种不同的代理函数: 高 斯过程(Gaussian processes, GP)、随机森林(random forest, RF)回归、TPE 模型. 选择函数包括 EI、概率 改进法(probability improvement, PI). 表 6 中对比 几种模型函数和方法. 超参数设置为 $\lambda_{1}=0.0015$, $\lambda_{2}=0.0460$, 选择不同的代理模型对识别率有影 响, 与不同模型对比, TPE, EI 可以得到最高的识 别率. 本文采用的自适应超参数方法可以得到更 准确的权值, 且选择的模型倾向于在超参数值附 近集中，从而产生最低损失，花费更多时间评估有 希望的超参数值. 使用贝叶斯优化完成模型的超 参数自动调整, 贝叶斯优化对目标函数的评估较 少, 测试集上有更好的泛化性能.

表 6 不同代理模型对识别率影响

\begin{tabular}{ccc}
\hline 代理函数 & 选择函数 & 识别率 $/ \%$ \\
\hline GP & PI & 93.34 \\
RF & PI & 93.56 \\
TPE & PI & 94.65 \\
GP & EI & 95.45 \\
RF & EI & 95.21 \\
TPE & EI & 96.27 \\
\hline
\end{tabular}

学习率分别设置为 $0.001,0.010, \delta$ 分别设置 为 $0.10,0.01$, 对超参数进行单约束和双约束, 得 到如表 7 所示相应的识别率. 由表 7 可以看出, 学 习率的选择对识别率有影响, 学习率小会导致特 征学习不充分, 会陷人局部最优, 0.010 的学习率 对模型的训练更好, 识别率得到提升; $\delta$ 是 Pseudo-Huber 损失函数的系数, 体现了对异常值 的处理能力, 根据对比可得出选取 0.010 的 $\delta$ 对异 常值处理效果好, 不会因为异常值的突变性影响 识别率, 针对日照强度条件提出的鲁棒特征损失 函数均使识别率有提高; 而与 Pseudo-Huber 损失

表 7 超参数对识别率的影响

\begin{tabular}{ccccc}
\hline 学习率 & $\delta$ & $\lambda_{1}$ & $\lambda_{2}$ & 识别率/\% \\
\hline 0.001 & 0.01 & & & 85.2 \\
0.010 & 0.01 & & & 88.5 \\
0.010 & 0.10 & & & 86.1 \\
0.010 & 0.01 & 0.0015 & & 91.3 \\
0.010 & 0.01 & & 0.046 & 93.8 \\
0.010 & 0.01 & 0.0015 & 0.046 & 95.4 \\
\hline
\end{tabular}

函数相比, 根据日照、对比度、平滑度和熵值提出 的灰度直方图统计矩缩小不同日照强度图像特征 差异的鲁棒损失函数, 进一步提高了识别率; 而在 联合约束下并选择最佳贝叶斯超参数的舰船识别率 达到 $95.00 \%$, 效果显著. 其中, 当 $\lambda_{1}=0.0015, \lambda_{2}=$ 0.0460 时, 训练识别率和验证识别率如图 5 所示.

图 5 所示为网络损失曲线和训练/验证识别率 曲线, 训练时网络损失快速收玫, 到 10000 步逐步 趋于稳定, 小幅度波动, 到 20000 步基本稳定于 0.0012. 训练识别率在 2000 步之前迅速提升, 在 达到 2000 步之后稳步波折式上升, 最终稳定在 95.00\%左右; 验证识别率在 10000 步时快速达到 $83.00 \%$ ，之后缓慢上升稳定到 $90.00 \%$. 所以，对 于不同日照强度样本的训练平均识别率达到 95.00\%, 验证识别率达到 $90.00 \%$, 对不同变化程 度的舰船图像都有着较高的识别效果.
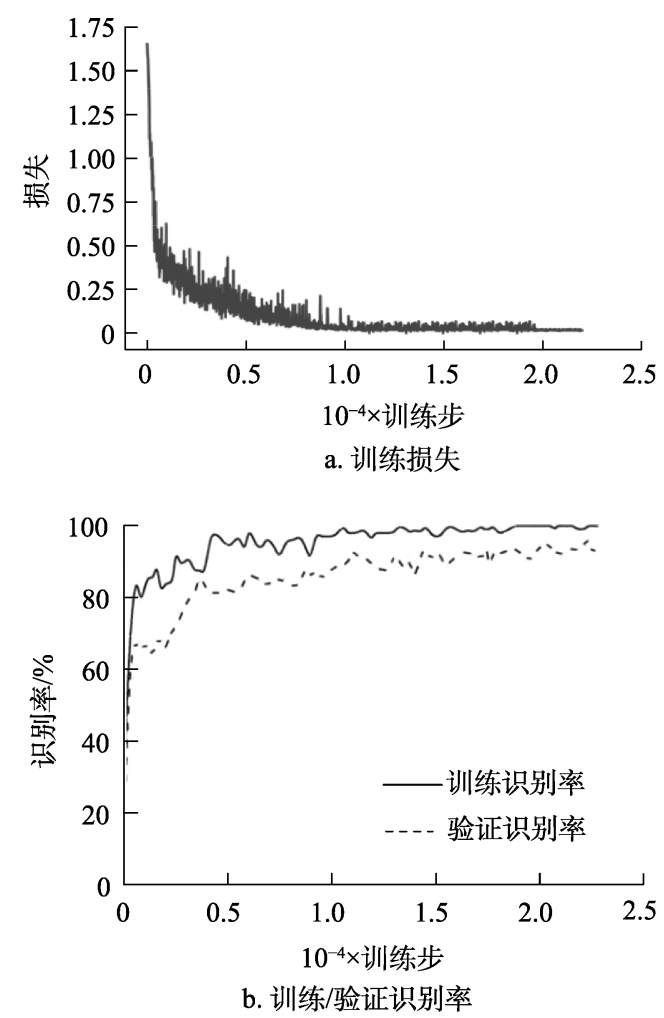

图 5 训练损失和训练/验证识别率

\section{2 识别率分析}

为验证不同日照强度条件下本文算法的识别 率, 分别与 NSCT+Lenet ${ }^{[22]}$, InceptionV3-FC ${ }^{[8]}$, ResNet- $50^{[16]}$, 多层 PCA 特征融合算法 ${ }^{[10]}, \mathrm{LBP}+\mathrm{DBN}^{[23]}$, Log-Gabor+LBP+DBN ${ }^{[24]}$ 以及新组合不变矩算法 进行对比，选取不同日照强度的舰船图像各 500 幅, 测试得到的平均识别率曲线, 如图 6 所示. 


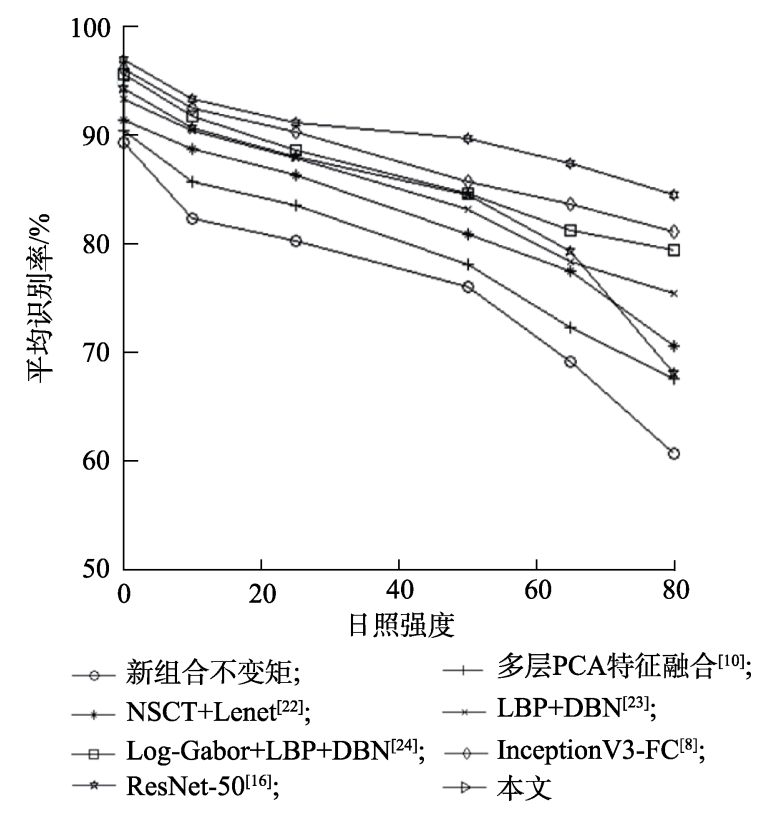

图 6 不同日照强度下各类算法识别率对比

从图 6 可以看出, 当日照强度逐渐加大时, 各 算法网络的平均识别率均下降. 当日照强度程度 为 $0 \sim 25$ 时, 本文算法识别率都能保持在 $90.00 \%$ 以 上; 日照强度为 $0 \sim 10,65 \sim 80$ 时, 识别率的下降速 度较快, 波动程度大, 特征的缺失会导致网络提取
的特征信息不完善，对舰船类别的分类影响大，识 别率会变低. 本文算法对日照强度 80 的样本达到 84.47\%的识别率, 与目前主流的传统算法、深度信 念网络结合的算法、融合算法以及新组合不变矩算 法进行对比, 本文算法的平均识别率为 $90.47 \%$.

表 8 所示为不同算法在不同日照强度下的平 均识别率分析对比. 其中, 新组合不变矩的识别率 最低, 多层 PCA 特征融合算法比组合不变矩有所 提高, 平均识别率提高了 3.40 个百分点; NSCT+ Lenet 算法比多层 PCA 特征融合算法提高了 1.51 个百分点, 其使用浅层的神经网络, 特征提取能力 比 Inception 和 ResNet 低, 故影响了识别率; 使用 深度信念网络与 Log-Gabor 滤波器和 LBP 特征方 法平均识别率比多层 PCA 特征融合算法提高了 3.45 个百分点, 网络的泛化能力较强; Log-Gabor+ $\mathrm{LBP}+\mathrm{DBN}$ 未考虑损失函数的约束导致识别率并 未有大的提高; InceptionV3-FC 使用 Logcos 损失函 数约束不同日照强度图像特征, 识别率达到了 $88.21 \%$; 而本文算法不仅考虑了损失函数对特征 的影响, 并针对日照提出统计矩约束, 故识别率 最高.

表 8 不同算法在不同日照强度情况下的平均识别率对比

\begin{tabular}{lccccccc}
\hline \multirow{2}{*}{\multicolumn{1}{c}{ 算法 }} & \multicolumn{7}{c}{ 日照强度 } \\
\cline { 2 - 8 } & 0 & 10 & 25 & 50 & 65 & 80 & 平均识别率/\% \\
\hline 新组合不变矩 & $89.31 \pm 0.12$ & $82.26 \pm 0.07$ & $80.21 \pm 0.06$ & $76.06 \pm 0.09$ & $69.18 \pm 0.08$ & $60.69 \pm 0.17$ & $78.28 \pm 0.19$ \\
多层 PCA 特征融合 $^{[10]}$ & $90.36 \pm 0.23$ & $85.65 \pm 0.12$ & $83.49 \pm 0.07$ & $78.03 \pm 0.06$ & $72.29 \pm 0.23$ & $67.61 \pm 0.07$ & $81.68 \pm 0.16$ \\
NSCT+Lenet & $91.35 \pm 0.18$ & $88.74 \pm 0.14$ & $86.25 \pm 0.23$ & $80.86 \pm 0.12$ & $77.42 \pm 0.09$ & $70.64 \pm 0.23$ & $83.19 \pm 0.07$ \\
LBP+DBN $^{[23]}$ & $93.24 \pm 0.07$ & $90.45 \pm 0.06$ & $87.86 \pm 0.12$ & $83.19 \pm 0.07$ & $78.35 \pm 0.12$ & $75.46 \pm 0.11$ & $85.16 \pm 0.06$ \\
Log-Gabor+LBP+DBN $^{[24]}$ & $95.59 \pm 0.14$ & $91.78 \pm 0.18$ & $88.61 \pm 0.14$ & $84.64 \pm 0.18$ & $81.28 \pm 0.15$ & $79.35 \pm 0.12$ & $86.64 \pm 0.12$ \\
InceptionV3-FC & $96.09 \pm 0.09$ & $92.46 \pm 0.15$ & $90.32 \pm 0.18$ & $85.66 \pm 0.14$ & $83.62 \pm 0.11$ & $81.12 \pm 0.18$ & $88.21 \pm 0.07$ \\
ResNet-50 & $94.27 \pm 0.15$ & $90.61 \pm 0.06$ & $88.02 \pm 0.09$ & $84.43 \pm 0.12$ & $79.28 \pm 0.23$ & $68.06 \pm 0.11$ & $88.96 \pm 0.12$ \\
本文 & $96.89 \pm 0.06$ & $93.28 \pm 0.11$ & $91.16 \pm 0.15$ & $89.64 \pm 0.12$ & $87.35 \pm 0.15$ & $84.47 \pm 0.09$ & $90.47 \pm 0.14$ \\
\hline
\end{tabular}

\section{3 类内间距和类间间距分析}

在舰船识别过程中, 同类舰船也会出现较大 的特征差异, 不同类舰船可能特征近似, 会出现误 判, 所以衡量类间距是识别的重要指标. 类间距和 类内距离可以体现出同种类别的共同性以及不同 类别的差异性, 类内散布矩阵 ${ }^{[25]}$ 表示各个样本围 绕均值的散布情况, 类间散布矩阵表示不同类别 在空间的散布情况，从 Softmax 损失的监督下训练 的深度神经网络中提取的特征是可分离的, 网络 的最后一个全连接层相当于线性分类器, 不同类 的深度特征通过决策边界被区分. 提取最后一层 网络输出的二维特征, 将特征空间可视化, 如图 7
所示. 由图 7 可知, 未经过特征约束的图像, 由于 日照因素以及不同类别之间的相似性，类间距离 较小, 类内距离较大, 越靠近中心, 不同种类的舰 船相似性越大, 样本分类模糊; 而经过约束之后的 舰船图像类内间距缩小, 类间距离扩大, 中心样本 分类较清晰, 这有助于舰船图像的正确分类, 提高 了识别率.

选取不同日照强度 $\psi=\{0,10,25,50,65,80\}$ 的舰 船图像各 500 幅, 计算经过全连接层后特征向量不 同舰船类别类内散布矩阵和类间散布矩阵的迹, 如表 9 所示. 由表 9 可以看出, 新组合不变矩算法 的类内距离最大, 类间距离最小, 这就导致在识别 

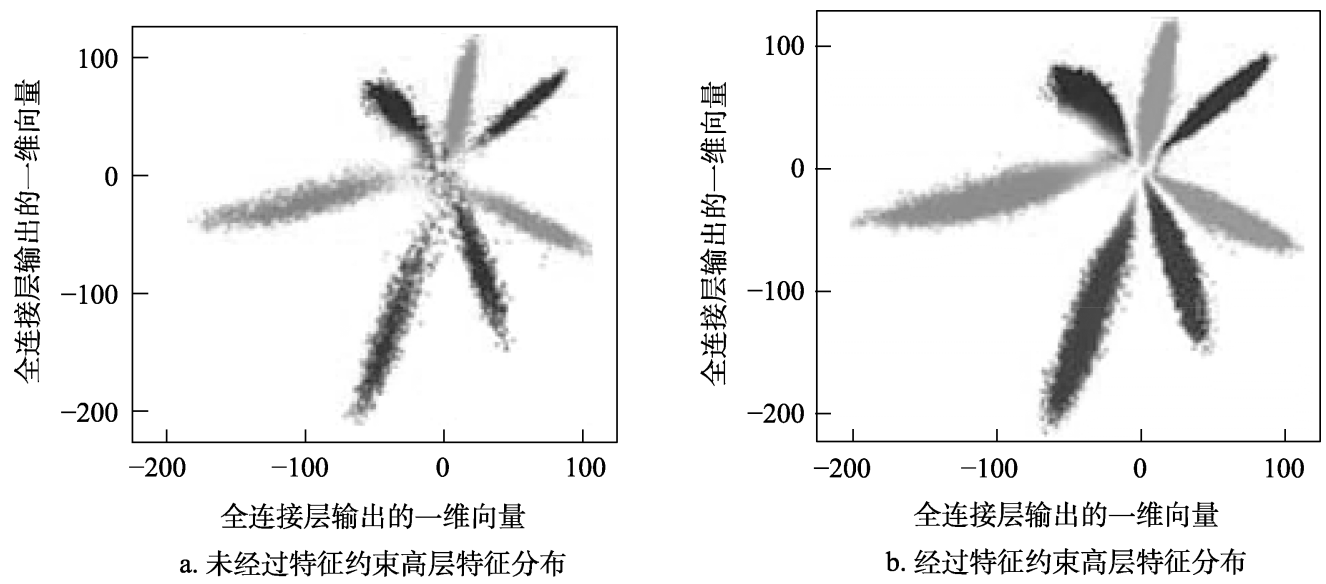

图 7 Softmax 监督下高层特征分布

过程中不同类别的舰船目标识别错误; Log-Gabor+ LBP 方法比 NSCT+Lenet算法有类间距离的控制有 所提高, 区分了不同类的差异性; InceptionV3-FC 方法减小了类内距离, 因为其损失函数约束了同 种类别舰船目标不同遮挡程度的差异; 本文算法 的类内距离最小，保证了同类目标的相似性，类间 距也最大, 使不同类别的目标差异性增大.

表 9 不同算法的类内距离和类间距离的迹对比

\begin{tabular}{|c|c|c|}
\hline 算法 & 类内距离的迹 & 类间距离的迹 \\
\hline 新组合不变矩 & 8.69 & 4.19 \\
\hline 多层 PCA 特征融合 ${ }^{[10]}$ & 7.95 & 4.83 \\
\hline NSCT+Lenet ${ }^{[22]}$ & 7.01 & 5.98 \\
\hline $\mathrm{LBP}+\mathrm{DBN}^{[23]}$ & 5.94 & 6.25 \\
\hline Log-Gabor+LBP+DBN ${ }^{[24]}$ & 4.26 & 8.76 \\
\hline InceptionV3-FC ${ }^{[8]}$ & 2.69 & 8.99 \\
\hline ResNet-50 $0^{[16]}$ & 2.21 & 9.38 \\
\hline 本文 & 1.06 & 10.65 \\
\hline
\end{tabular}

\section{4 结 语}

针对日照强度因素对舰船目标识别率的影响, 本文算法取得了良好成效，在实验训练测试以及 算法对比中都比其他算法的识别率高. 本文对日 照强度变化前后特征使用 Pseudo-Huber 损失约束, 并计算特征灰度直方图的统计矩约束光照因素影 响, 贝叶斯自适应超参数优化比人工调节权重简 便很多, 而且选择最佳权重提高了识别率. 从表 8 可以看出，本文算法对改进前不同日照强度的识 别率均有提高, 日照强度为 $0,10,25,50,65,80$ 的 识别率提高的百分点分别为 $2.62,2.67,3.14,5.21$, 8.07，16.41. 识别算法可应用在侦察取证、军事领 域敌情侦察、机器人船舶导航、导弹导航制导、海
上救援等方面. 本文着重考虑了日照因素对舰船 识别的影响, 并取得了不错的效果, 但未考虑云雾 变化、低照度图像，或者礁石、舰船等现实遮挡物 的影响, 后续将改进实验. 对于自适应学习率的探 讨、Softmax 改进分类函数、中心损失的衡量、使 用滤波器和 LBP 特征级联约束等都是可以继续研 究的话题.

\section{参考文献(References):}

[1] Peng Jingtao. The study of ship target detection in optical remote sensing images[D]. Shanghai: Donghua University, 2017(in Chinese)

(彭敬涛. 光学遥感图像舰船目标检测技术的研究[D]. 上海: 东华大学, 2017)

[2] Wang Huili, Zhu Ming, Lin Chunbo, et al. Ship detection of complex sea background in optical remote sensing image[J]. Optical Precision Engineering, 2018, 26(3): 723-732(in Chinese)

(王慧利, 朱明, 萄春波, 等. 光学遥感图像中复杂海背景下 的舰船检测 [J]. 光学精密工程, 2018, 26(3): 723-732)

[3] Wang H L, Zhu M, Lin C B, et al. Ship detection in optical remote sensing image based on visual saliency and AdaBoost classifier[J]. Optoelectronics Letters, 2017, 13(2): 151-155

[4] Zhang Y, Lei Z Y, Zhuang L, et al. A CNN based method to solve class imbalance problem in SAR image ship target recognition[C] //Proceedings of the 5th Advanced Information Technology, Electronic and Automation Control Conference. Los Alamitos: IEEE Computer Society Press, 2021: 229-233

[5] Krizhevsky A, Sutskever I, Hinton G E. ImageNet classification with deep convolutional neural networks[J]. Communications of the ACM, 2017, 60(6): 84-90

[6] Liu Feng, Shen Tongsheng, Ma Xinxing. Convolutional neural network based multi-band ship target recognition with feature fusion[J]. Acta Optica Sinica, 2017, 37(10): 248-256(in Chinese)

(刘峰, 沈同圣, 马新星. 特征融合的卷积神经网络多波段 
舰船目标识别[J]. 光学学报, 2017, 37(10): 248-256)

[7] Zou Z X, Shi Z W. Ship detection in spaceborne optical image with SVD networks[J]. IEEE Transactions on Geoscience and Remote Sensing, 2016, 54(10): 5832-5845

[8] Cheng G, Zhou P C, Han J W. Learning rotation-invariant convolutional neural networks for object detection in VHR optical remote sensing images[J]. IEEE Transactions on Geoscience and Remote Sensing, 2016, 54(12): 7405-7415

[9] Wu Shukai, Liu Baolong, Xu Shuchang, et al. A two-stage ship license plate locating algorithm based on deep feature transfer and fusion[J]. Journal of Computer-Aided Design \& Computer Graphics, 2020, 32(4): 628-634(in Chinese)

(吴书楷, 刘宝龙, 徐舒畅, 等. 结合深度特征迁移与融合的 两阶段船牌定位算法 [J]. 计算机辅助设计与图形学学报, 2020, 32(4): 628-634)

[10] Liu Haobo, Shi Yuexiang. Face occlusion detection based on multi-layer PCA feature fusion[J]. Journal of Hunan City University: Natural Science Edition, 2018, 27(1): 43-47(in Chinese)

(刘浩博, 石跃祥. 基于多层 PCA 特征融合的人脸遮挡检测 [J]. 湖南城市学院学报: 自然科学版, 2018, 27(1): 43-47)

[11] Liu Huan, Hao Kuangrong, Ding Yongsheng, et al. An image matching method of new illumination-robust and anti-blur combined moment invariants[J]. Journal of Sensor Technology, 2013, 26(9): 1258-1264(in Chinese)

(刘欢, 郝矿荣, 丁永生, 等. 光照鲁棒的抗模糊新组合不变 矩图像匹配方法[J]. 传感技术学报, 2013, 26(9): 1258-1264)

[12] Zhuang Y Y, Guan Y P. Deep learning for face recognition under complex illumination conditions based on log-Gabor and LBP[C] //Proceedings of the 3rd Information Technology, Networking, Electronic and Automation Control Conference. Los Alamitos: IEEE Computer Society Press, 2019: 1926-1930

[13] Huang Jie, Jiang Zhiguo, Zhang Haopeng, et al. Ship object detection in remote sensing images using convolutional neural networks[J]. Journal of Beijing University of Aeronautics and Astronautics, 2017, 43(9): 1841-1848(in Chinese) (黄洁, 姜志国, 张浩鹏, 等. 基于卷积神经网络的遥感图像 舰船目标检测 [J]. 北京航空航天大学学报，2017，43(9): 1841-1848)

[14] Qi Chao, Wang Xiaofeng. Transport ships recognition method based on convolution neural networks[J]. Microcomputer and Application, 2017, 36(17): 52-55(in Chinese) (戚超, 王晓峰. 基于卷积神经网络的运输船舶分类识别方 法[J]. 微型机与应用, 2017, 36(17): 52-55)

[15] Di L, Long H B, Liang J Z. Fabric defect detection based on illumination correction and visual salient features[J]. Sensors, 2020, 20(18): 5147
[16] He K M, Zhang X Y, Ren S Q, et al. Deep residual learning for image recognition[C] //Proceedings of the IEEE Conference on Computer Vision and Pattern Recognition. Los Alamitos: IEEE Computer Society Press, 2016: 770-778

[17] Wang Youming, Xu Panfeng. CNN electron microscope images classification based on improved Adam optimizer[J]. Journal of Xi'an University of Posts and Telecommunications, 2019, 24(5): 26-33(in Chinese)

(汪友明, 徐攀峰. 基于改进 Adam 优化器的 CNN 电镜医学 图像分类 [J]. 西安邮电大学报, 2019, 24(5): 26-33)

[18] Cai X, Xue L G, Lu F. Robust estimation with a modified Huber's loss for partial functional linear models based on splines[J]. Journal of the Korean Statistical Society, 2020, 49(3): 1214-1237

[19] Zhu Huilong, Liu Xiaoyan, Liu Yao. Research on hyperparameter optimization of new deep learning based on Bayes[J]. Data Communications, 2019(2): 35-38+46(in Chinese)

(朱汇龙, 刘晓燕, 刘瑶. 基于贝叶斯新型深度学习超参数 优化的研究 [J]. 数据通信, 2019(2): 35-38+46)

[20] Aouat S, Ait-Hammi I, Hanmouchene I. A new approach for texture segmentation based on the gray level co-occurrence matrix[J]. Multimedia Tools and Applications, 2021, 80: 24027-24052

[21] van Molle P, Verbelen T, Vankeirsbilck B, et al. Leveraging the Bhattacharyya coefficient for uncertainty quantification in deep neural networks[J]. Neural Computing and Applications, 2021, 33: 10259-10275

[22] Zhao Z H, Liang J L. NSCT-based adaptive illumination processing for face recognition[C] //Proceedings of the International Conference on Information Systems and Computer Aided Education. Los Alamitos: IEEE Computer Society Press, 2018: 218-221

[23] Dandpat S K, Meher S, Bopche V. Uneven illumination compensation for unconstrained face recognition using LBP $[\mathrm{C}]$ //Proceedings of the 3rd International Conference for Convergence in Technology. Los Alamitos: IEEE Computer Society Press, 2018: 1-6

[24] Lin H N, Shi Z, Zou Z. Fully convolutional network with task partitioning for inshore ship detection in optical remote sensing images[J]. IEEE Geoscience and Remote Sensing Letters, 2017, 14(10): 1665-1669

[25] Zhang Suzhi, Chen Xiaoni, Yang Rui, et al. Method of principal component analysis based on intra-class distance and inter-class distance[J]. Computer Engineering and Design, 2020, 41(8): 2177-2183(in Chinese) (张素智, 陈小妮, 杨芮, 等. 基于类内和类间距离的主成分 分析算法[J]. 计算机工程与设计, 2020, 41(8): 2177-2183) 